\title{
CONVERGENCIA ECONÓMICA EN LOS DEPARTAMENTOS DE MENDOZA
}

\author{
Elizabeth Pasteris* \\ Tomás Kemnitz* \\ Franco Bignone*
}

enviado: abril 2015 - aceptado: febrero 2016

\begin{abstract}
Resumen
En este trabajo se analiza la convergencia económica de los departamentos de Mendoza durante el período 1996 a 2012. Se examina la evolución de las desigualdades territoriales mediante indicadores de disparidad, dispersión y brechas de crecimiento. No se encuentran evidencias de un proceso de convergencia territorial. El coeficiente $\beta$ estimado resulta positivo y estadísticamente no significativo; el Coeficiente de Williamson, $\sigma$-convergencia y brechas de crecimiento revelan un leve aumento de la desigualdad, especialmente durante períodos de auge. Tampoco se encontró evidencia de relación positiva entre crecimiento y especialización agropecuaria. La inserción internacional del sector agroindustrial no contribuyó a mejorar significativamente la situación de los territorios fuertemente agropecuarios, los de menor desarrollo relativo provincial.
\end{abstract}

Código JEL: R1, O1.

Palabras clave: convergencia económica, economía regional, ordenamiento del territorio, desarrollo local.

* Universidad Nacional de Cuyo, Mendoza, Argentina. Facultad de Ciencias Económicas. Centro Universitario s/n- Mendoza, Argentina. Correos electrónicos: lizzipasteris@gmail.com; tomaskemnitz@yahoo.com.ar; franco_bignone@hotmail.com 


\begin{abstract}
This article looks at the evidence of economic convergence across Mendoza departments from 1996 to 2012. The evolution of regional inequality is analyzed through disparity and dispersion indices and growth gaps. No evidence of territorial convergence has been found. The estimated $\beta$ coefficient is positive and statistically not significant; Williamson's coefficient, $\sigma$-convergence and growth gaps show a slight inequality increase, particularly during booms. Similarly, no evidence of a positive relation between agricultural specialization and growth has been found. In fact, our results suggest that the agricultural territories, i. e., the comparatively less-developed ones, do not exhibit an improvement in their relative position, despite the favorable international context.
\end{abstract}

JEL Code: R1, O1.

Keywords: regional economics, economic convergence, local development, territorial planning.

\title{
INTRODUCCIÓN
}

La provincia de Mendoza ha experimentado un aceptable crecimiento en las últimas décadas, siguiendo la evolución económica nacional, pero también apoyada en las exportaciones, que han dinamizado la economía provincial. En efecto, entre 2003 y 2005 el nivel de actividad económica creció más rápidamente que las exportaciones, evidenciado recuperación del mercado interno. En adelante, las ventas al exterior tuvieron un dinamismo superior al del PBG. Este proceso se vio interrumpido en el año 2009 , debido fundamentalmente a la crisis internacional, con una contracción del $4 \%$ de los montos exportados, situación que se revirtió en el año 2010, presentándose a partir de entonces un crecimiento oscilante. En síntesis, entre 2003 y 2012, las exportaciones de Mendoza se duplicaron, impulsadas fundamentalmente por las manufacturas de origen agropecuario.

También se produjo una importante modificación en la composición de la matriz exportadora provincial, con disminución en la participación de combustibles y crecimiento de las manufacturas de origen agropecuario, que representaron en el año 2013 el $68 \%$ del total ${ }^{1}$. Entre ellas, las exportaciones de la cadena vitivinícola ocupan un lugar destacado.

Su participación era del 34\% en el año 2003. 
Por otra parte, más allá de las características que permiten definir a una provincia como unidad político-institucional y económica en sí misma, es posible profundizar el análisis e indagar acerca de las diversidades y singularidades que presenta su interior. En efecto, dentro de Mendoza coexisten diferentes realidades según se consideren las contingencias climáticas, la disponibilidad hídrica, el relieve, la matriz productiva histórica, etc.

Figura 1. Mendoza. PBG per cápita. En miles de pesos de 1993

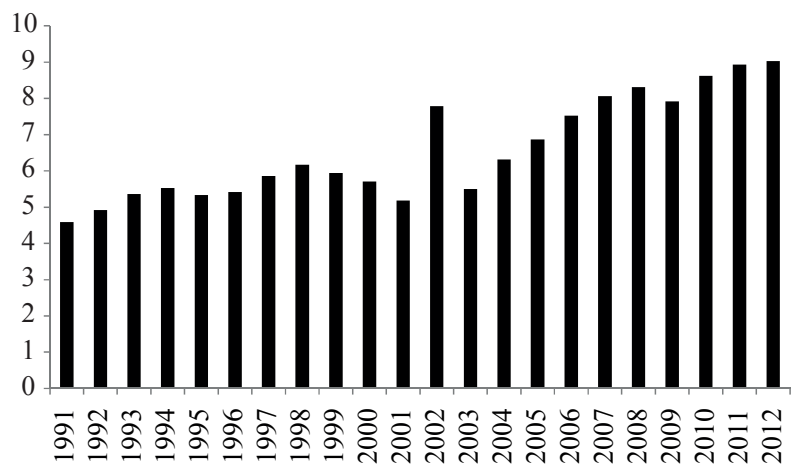

\begin{tabular}{ccccccccccc}
\hline $\mathbf{1 9 9 1}$ & $\mathbf{1 9 9 2}$ & $\mathbf{1 9 9 3}$ & $\mathbf{1 9 9 4}$ & $\mathbf{1 9 9 5}$ & $\mathbf{1 9 9 6}$ & $\mathbf{1 9 9 7}$ & $\mathbf{1 9 9 8}$ & $\mathbf{1 9 9 9}$ & $\mathbf{2 0 0 0}$ & $\mathbf{2 0 0 1}$ \\
\hline 4,5916 & 4,9201 & 5,3622 & 5,5267 & 5,332 & 5,4181 & 5,8593 & 6,1665 & 5,9403 & 5,7092 & 5,1824 \\
\hline $\mathbf{2 0 0 2}$ & $\mathbf{2 0 0 3}$ & $\mathbf{2 0 0 4}$ & $\mathbf{2 0 0 5}$ & $\mathbf{2 0 0 6}$ & $\mathbf{2 0 0 7}$ & $\mathbf{2 0 0 8}$ & $\mathbf{2 0 0 9}$ & $\mathbf{2 0 1 0}$ & $\mathbf{2 0 1 1}$ & $\mathbf{2 0 1 2}$ \\
\hline 4,7886 & 5,4993 & 6,3157 & 6,8735 & 7,5253 & 8,0614 & 8,3097 & 7,9147 & 8,6211 & 8,9298 & 9,0304 \\
\hline
\end{tabular}

Fuente: elaboración propia sobre DEIE y FCE-UNCuyo.

Reconociendo estas circunstancias, el objetivo de este trabajo es avanzar en el conocimiento de la distribución de la renta en el territorio. Como destaca Easterly (2001), "la reducción en variables como el hambre, la mortalidad y la pobreza a medida que el $\mathrm{PBI}$ per cápita aumenta es lo que motiva los estudios sobre las claves del crecimiento". En este punto, se genera un interrogante casi forzosamente: ¿están las regiones pobres destinados a permanecer como tales? $\mathrm{O}$, por el contrario, ¿se verifica una tendencia de disminución de las diferencias de la renta per cápita entre regiones ricas y pobres? Esto último se conoce como hipótesis de convergencia económica y es lo que trata de probarse en este artículo. 
Figura 2. Evolución de las exportaciones de Mendoza.

En millones de dólares

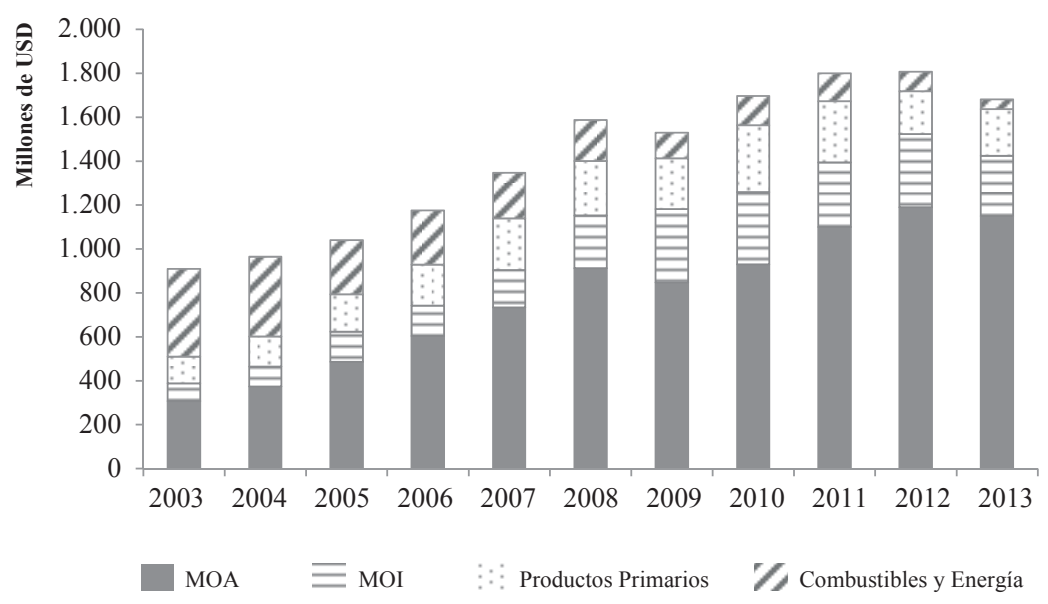

Fuente: elaboración propia con base en INDEC.

Como hipótesis de trabajo se plantea, además, que la coyuntura macroeconómica nacional, que favoreció a los sectores agroexportadores provinciales a lo largo de casi toda la última década, benefició a las regiones menos desarrolladas de la provincia, que tienen como principal actividad económica la producción agraria, contribuyendo así a la disminución en la brecha regional.

\section{LA TEORÍA}

\section{I.1. Marco teórico}

El crecimiento es un fenómeno que despertó interés en los estudiosos desde el nacimiento de la economía clásica, con David Ricardo, Adam Smith y Thomas Malthus, pero comenzó a ser analizado con mayor disciplina a principios del siglo $\mathrm{XX}$, cuando se comenzaron a formalizar los primeros modelos de crecimiento. Más específicamente, uno de los fenómenos que ha obtenido sostenida atención en los últimos años es el de la convergencia económica. Los modelos neoclásicos, entre los cuales el de Solow-Swan es el más reconocido, predicen que las diferencias en el ingreso per cápita entre distintas economías surgen, al menos, por tres razones: 
- Por un lado, porque las economías tienden naturalmente a su estado estacionario. Si las diferencias en el ingreso per cápita surgen porque los países están en diferentes puntos con respecto a su estado estacionario, es de esperar que las economías pobres crezcan más que las ricas.

- En segundo lugar, el modelo neoclásico de crecimiento implica que la tasa de rendimiento del capital es menor en países con mayor nivel de capital por habitante. Esto indica que existen incentivos para que el capital fluya desde los países ricos hacia los pobres; hecho que afianza la hipótesis de convergencia.

- Finalmente, si existen rezagos en la difusión del conocimiento, las diferencias de ingreso pueden surgir porque algunos países no están empleando las mejores tecnologías disponibles. Estas diferencias podrían tender a desaparecer a medida que las economías más pobres van ganando acceso a mejores métodos y tecnologías de producción.

Específicamente, si se supone una función de producción que depende de capital, trabajo y conocimiento (también interpretado como "rendimiento del trabajo"), con rendimientos constantes a escala de tipo Cobb-Douglas, productividades marginales decrecientes en los factores de producción, y tasas de crecimiento constantes en el tiempo para el conocimiento y la población, se obtiene la ecuación fundamental del modelo²:

$$
\dot{k} / k=s k^{\alpha-1}-(n+g+\delta)
$$

Donde $k$ es el capital per cápita de la economía, $S$ es la proporción del producto que se destina a inversión (constante y exógena), $\alpha$ es la participación del factor de producción capital en el ingreso total, $n$ es la tasa de crecimiento de la población, $g$ es la tasa de crecimiento del conocimiento y $\delta$ es la tasa de depreciación del capital. El punto por encima de la variable es la derivada de con respecto al tiempo (que se considera continuo).

La ecuación anterior muestra la tasa de crecimiento del capital per cápita, la cual se puede demostrar que es igual a la tasa de crecimiento del ingreso per cápita. La representación gráfica de esta ecuación permite comprender el concepto de convergencia con mayor claridad:

2 Con base en Romer, D. (1996). 
Figura 3. Ecuación fundamental del modelo de Solow

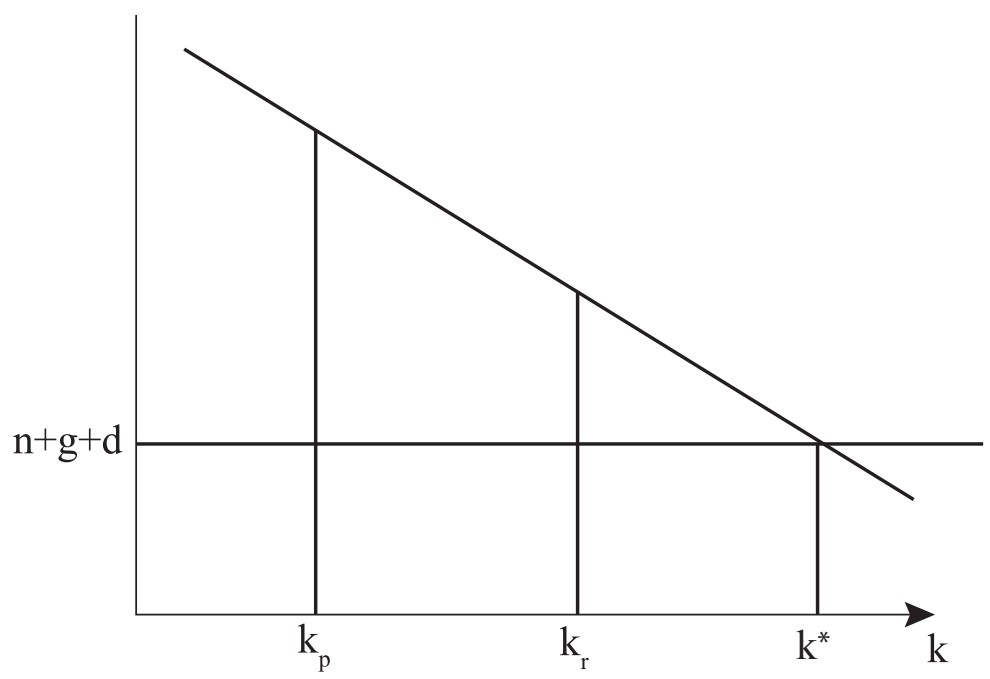

Fuente: elaboración propia.

La idea central de este proceso es que la función de producción tiene rendimientos constantes a escala, pero rendimientos decrecientes en el factor que se puede acumular. En la figura anterior, la curva con pendiente negativa representa el primer término del lado derecho de la ecuación y la línea horizontal es la suma de $n+g+d$. La tasa de crecimiento es la diferencia vertical entre estas dos curvas. En este contexto, se supone que existen dos economías, una rica (caracterizada por un alto nivel de capital per cápita inicial, $\mathrm{K}^{*}$ ) y una pobre (con un nivel inicial más bajo, $\mathrm{K}_{\mathrm{p}}$ ). Se observa que la tasa de crecimiento es mayor en la región pobre que en la rica y que esta tasa es decreciente en el tiempo (Porto, 1994).

\section{I.2. Antecedentes}

La convergencia obtenida en los modelos neoclásicos ha sido objeto de análisis empírico durante muchos años. El concepto ha sido desglosado en dos categorías, desarrolladas inicialmente por Sala-i-Martín (1990): $\beta$-convergencia y $\sigma$-convergencia. Existe $\beta$-convergencia si las economías pobres crecen más que las ricas en un lapso determinado; en otras palabras, si existe una relación inversa entre la tasa de crecimiento de la renta y el nivel inicial de la misma. Este concepto se confunde a menudo con el de $\sigma$-convergencia. Se dice que existe 
$\sigma$-convergencia cuando la dispersión de la renta real per cápita entre grupos de economías tiende a reducirse en el tiempo ${ }^{3}$.

En la década del ochenta, Sala-i-Martín realizó el primer estudio para analizar estas dos categorías de convergencia con un conjunto de datos comparables de PIB real de ciento catorce países, para el período 1960-1985. Demostró que no existía una relación negativa entre la tasa de crecimiento de cada país y el ingreso per cápita del año inicial, con lo cual descartó la existencia de $\beta$-convergencia. Por otra parte, la dispersión del PIB per cápita aumentó durante el período, derribando la hipótesis de la existencia de la $\sigma$-convergencia. Inicialmente esto se tomó como evidencia a favor de los modelos de crecimiento endógeno, en detrimento de los modelos neoclásicos de rendimientos decrecientes del capital. El aparente fracaso empírico de los modelos neoclásicos fue una de las causas determinantes del extraordinario éxito que los modelos endógenos cosecharon durante las décadas ochenta y noventa.

A principio de los años noventa, economistas como Sala-i-Martín, Barro, Mankiw, Romer y Weil comenzaron una contundente defensa de los modelos neoclásicos, argumentando que los mismos predicen que la tasa de crecimiento de una economía está inversamente relacionada con la distancia que la separa de su propio estado estacionario. Solo si todas las economías se acercan al mismo estado estacionario esta afirmación equivale a sostener que las economías pobres crecerán más que las ricas. En el contexto del modelo de Solow, las regiones deberían presentar idénticas tasas de ahorro, tecnología, depreciación y crecimiento de la población para que la existencia de convergencia resultara equivalente a mayor tasa de crecimiento de las economías pobres (respecto de las ricas).

Estos economistas desarrollaron el concepto de "convergencia condicional" para contraponerlo al de "convergencia absoluta" utilizado hasta el momento. Hasta entonces se habían realizado regresiones teniendo en mente el concepto de convergencia absoluta, ya que implícitamente se estaba considerando que todas las economías de la muestra eran iguales (en preferencias, tecnología e instituciones) y por lo tanto debían tender a un mismo estado estacionario. Claramente este supuesto no tiene ningún tipo de sustento empírico y por lo tanto no se puede considerar que la falta de convergencia absoluta entre en contradicción con el modelo neoclásico de crecimiento. Para hacer el test del modelo neoclásico, había que mejorar el concepto de convergencia absoluta y lograr medir de alguna manera

3 Puede demostrarse que la existencia de $\beta$-convergencia es una condición necesaria, pero no suficiente para la existencia de $\sigma$-convergencia. 
la distancia entre el nivel de renta de un país y el nivel de renta en su propio estado estacionario. En términos estadísticos, había que encontrar una correlación parcial negativa entre nivel de renta inicial y crecimiento, condicional al estado estacionario de cada economía.

Empíricamente, hay dos maneras de condicionar los datos: a) limitar el estudio a conjuntos de economías parecidas, en el sentido de que están pobladas por individuos con preferencias similares, con instituciones y sistemas impositivos y legales parecidos y empresas que se enfrentan a funciones de producción parecidas. Si se cumplen estos supuestos, entonces se debería encontrar convergencia entre este grupo de economías, dado que todas ellas tenderán a acercarse al mismo estado estacionario. Un ejemplo de este tipo de condicionamientos podrían ser las regiones de un mismo país. b) condicionar los datos mediante la utilización de regresiones múltiples. La idea de este método es efectuar una regresión con datos de sección cruzada del crecimiento sobre la renta inicial, manteniendo constante un cierto número de variables adicionales (que actúan de proxy del estado estacionario). Si el resultado es que el coeficiente de la renta inicial es negativo, entonces existe $\beta$-convergencia condicional.

En 1992, Sala-i-Martín aplica el primer método de condicionamiento de los datos y realiza el análisis para los estados federales de Estados Unidos, las prefecturas japonesas y para las comunidades autónomas españolas. En todos los casos obtiene una relación negativa entre crecimiento e ingreso inicial, lo que denota la existencia de $\beta$-convergencia. En cuanto a $\sigma$-convergencia, también observa un descenso temporal de la dispersión en todos los países. Estos resultados fueron tomados por gran parte de la comunidad académica como una victoria de los modelos neoclásicos por sobre los de crecimiento endógeno.

En el caso argentino, el tema de la convergencia ha sido explorado por algunos investigadores como Porto (1994, 1995, 1996), Marina (1998), Utrera y Koroch (1998), Figueras Arrufat y Regis (2003) y Garrido, Marina y Sotelsek (2002). En la tabla 1 se presentan algunos de los resultados obtenidos.

Como se ve, se rechaza la hipótesis de $\beta$-convergencia en todos los casos y en aquellas investigaciones donde se estudió $\sigma$-convergencia sucede lo mismo. Es importante mencionar que los resultados para $\beta$-convergencia analizados fueron realizados a partir del primero de los métodos de condicionamiento de datos. Muchos autores eligen llamar a este análisis " $\beta$-convergencia absoluta" pese a no tratarse de la interpretación original de "absolutismo". Denominan " $\beta$-convergencia condicional" al método de regresiones múltiples. Con esta técnica estadística, 
varios de los investigadores (como Porto en algunos de sus tantos análisis o Utrera y Koroch [1998] y Figueras, Arrufat y Regis [2003]) demostraron la existencia de convergencia en el sentido $\beta$. Esto implicaría que la política económica "contamina" el proceso de convergencia a partir del sistema de coparticipación nacional vigente, del gasto público, la inversión estatal, etcétera.

Tabla 1. $\beta$ y $\sigma$ convergencia en las provincias argentinas

\begin{tabular}{lccc}
\hline Autor & Período & $\begin{array}{c}\beta- \\
\text { convergencia }\end{array}$ & $\begin{array}{c}\sigma- \\
\text { convergencia }\end{array}$ \\
\hline G. Porto (1994) & $1953-1980$ & Se rechaza & \\
Utrera y Koroch (1994) & $1953-1994$ & Se rechaza & Se rechaza \\
A. Marina (1998) & $1970-1995$ & Se rechaza & Se rechaza \\
Garrido, Marina y Sotelsek(2002) & $1970-1996$ & Se rechaza & \\
Figueras, Arrufat, Regis (2003) & $1980-1998$ & Se rechaza & \\
\hline
\end{tabular}

Fuente: elaboración propia sobre la base de los autores.

Siguiendo un esquema de razonamiento similar, Platino analiza el período 1993-2003. Concluye que

Santa Fe presenta un patrón de concentración industrial muy marcado que incluso se refuerza en el tiempo. Al complementar el análisis evaluando la convergencia económica, los resultados reflejan un proceso de mayor divergencia en los registros de actividad industrial entre los departamentos de Santa Fe (Platino, 2013).

Si bien no se ha observado un proceso de convergencia entre los niveles de ingreso por habitante entre las provincias de nuestro país en los distintos períodos analizados, Capello, Figueras, Freille y Moncarz demuestran que existió convergencia entre provincias respecto de indicadores de salud, educación, vivienda, seguridad y otros, que actúan como proxy del bienestar de la población, durante el período 1980-2001. De todos modos, no se puede asegurar que este resultado sea consecuencia del sistema de transferencias fiscales existente en el país, tal como lo establecía su hipótesis de investigación (Capello, Figueras, Freille \& Moncarz, 2011). 


\section{METODOLOGÍA}

Como se mencionó anteriormente, el objetivo principal de este trabajo es analizar la evolución de la desigualdad económica entre los departamentos que componen la provincia de Mendoza. Como eje central se estima y analiza la convergencia del Producto Bruto Geográfico per cápita (PBGpc) de los dieciocho departamentos, y de las regiones que los agrupan, durante el período 1996 a 2012. Esta metodología se sustenta en una extensa lista de antecedentes y, fundamentalmente, en el renombrado trabajo de Sala-i-Martín (ver sección I), que proponen una relación inversa entre tasa de crecimiento y nivel inicial de la renta. Si bien la convergencia es un fenómeno de largo plazo, por lo que contar con una serie más extensa hubiera sido, sin dudas, deseable, la elección del período fue impuesta por la disponibilidad de datos.

Por otro lado, con el objetivo de enriquecer el análisis de la evolución de las desigualdades, se agregan indicadores de disparidad, de dispersión y brechas de crecimiento, sobre la base de la metodología utilizada en Russo y Delgado (2000).

Finalmente, se estiman indicadores de especialización y concentración económica, que permiten interpretar los resultados obtenidos según su relación con la matriz productiva de cada territorio. Estos indicadores son la clave para relacionar crecimiento con especialización productiva departamental, lo cual, se espera, pueda ayudar a confirmar o rechazar la hipótesis principal de trabajo. Se eligieron los siguientes indicadores: Índice de Especialización Simple (IES), que se aplicó al sector agropecuario, y que muestra cuán especializado está un departamento en relación con el promedio provincial; Coeficiente de Especialización (CE), que muestra la cercanía entre la estructura productiva del territorio analizado y el promedio provincial e Índice de Herfindahl Hirschman, que indica cuán concentrada o diversificada está la estructura productiva del territorio en cuestión.

\section{II.1. Indicadores de disparidad}

Se recurrió al Coeficiente de Williamson (CW) para medir disparidad entre unidades territoriales. El CW es un coeficiente de variación del PBG por habitante ponderado por la importancia de cada territorio en el PBG provincial. Este indicador toma valor cero cuando la igualdad es máxima. Se calcula de la siguiente manera: 


$$
C W=\frac{\sqrt{\sum_{i=1}^{n}\left(y_{i}-\bar{y}\right)^{2} \frac{x_{i}}{\bar{x}}}}{\bar{y}}
$$

Donde $y_{i}$ es el ingreso per cápita del departamento $i, \bar{y}_{\text {es el ingreso per cápita }}$ provincial; $x_{i}$ es la población del departamento $i$ y $\bar{x}_{\text {la población provincial. }}$

\section{II.2. Indicadores de dispersión}

Como medida de dispersión se utiliza Convergencia Sigma, definida como:

$$
C v \sigma_{t}=\sqrt{\sum_{i=1}^{n}\left(\frac{L n P B G p c_{i t}-L n P B G p c t}{n}\right)^{2}}
$$

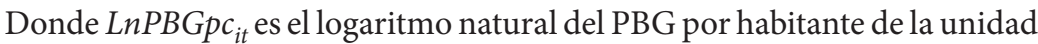
territorial $i$ en el año $t$; $L n P B G p c_{t}$ es el logaritmo natural del PBG por habitante promedio ponderado de todas las unidades territoriales en el año $t y$, finalmente, $n$ mide la cantidad de regiones o territorios.

\section{II.3. Brecha de crecimiento}

Para los períodos 1996-2003 y 2003-2012 se determinó, para cada unidad territorial, su tasa de crecimiento anual real (TCA), la tasa de crecimiento estimada (TCE) requerida para alcanzar el $P B G p c$ más elevado del final de cada período y la tasa de crecimiento promedio (TCP) necesaria para alcanzar el $P B G P C$ promedio de cada uno de los períodos. De esta forma se determinó la brecha que separa a cada unidad territorial de la que ocupa la mejor posición y del promedio provincial. Asimismo, siguiendo la metodología propuesta por Sala-i-Martín (2000), se añade la estimación de Convergencia Beta.

Cabe destacar que la fuerte interrelación entre departamentos vecinos, que hace que sus fronteras sean difusas, hace que sea posible que el $P B G p c$ no sea una medida realmente precisa del ingreso por habitante. Por ello, se complementan los resultados obtenidos con el análisis a nivel de regiones. En el futuro, este estudio podría ser comparado con el análisis de convergencia de indicadores sociales, que reflejen el bienestar. 


\section{II.4. Relación entre convergencia y especialización económica}

Con el fin de relacionar los resultados del estudio de convergencia con la estructura productiva de la provincia, se estimaron indicadores de especialización y concentración económica. Estos índices permiten describir las estructuras productivas territoriales y realizar comparaciones temporales y transversales. Se entiende que una economía se encuentra altamente especializada cuando es muy acotada la gama de bienes o servicios que se dedica a elaborar. De forma opuesta, se considera que una economía está poco especializada cuando es muy amplia la gama de productos o servicios que elabora. Además de calcular las participaciones sectoriales en el PBG de cada departamento, se procedió a calcular los siguientes indicadores:

- Coeficiente de especialización $(C E)$ : mide el grado de similitud de la estructura productiva de una zona respecto de un área de referencia. Su fórmula es la siguiente:

$$
C E_{j}=\frac{1}{2} \sum_{i=1}^{h}\left|\frac{x_{i j}}{x_{j}}-\frac{x_{i}}{x}\right|
$$

siendo $x_{i j}$ el valor agregado bruto $(V A B)$ del i-ésimo sector de la región $j$; $x_{\mathrm{j}}$ el VAB de la j-ésima región; $x_{i}$ el $V A B$ del i-ésimo sector del área de referencia; $x$ el $V A B$ de todos los sectores del área de referencia y $h$ los sectores considerados. Este coeficiente varía entre 0 y 1 . Si su valor es 0 , la región bajo análisis tiene la misma composición de actividades que el área de referencia. Lo contrario sucederá cuando el valor del coeficiente se aproxime a la unidad.

- Índice de especialización simple (IES): mide la especialización relativa de un territorio respecto de un área de referencia, para un sector en particular. Su cálculo consiste en comparar la participación del i-ésimo sector $\left(x_{i j}\right)$ en el VAB total de la j-ésima zona $\left(x_{j}\right)$ con esa misma participación en el aérea de referencia elegida:

$$
\text { IES }=\frac{x_{i j} / x_{j}}{x_{i} / x}
$$

De esta forma, se puede determinar si un territorio presenta mayor, igual o menor especialización que el área de referencia en un sector determinado. Si ambas participaciones son iguales el IES será igual a 1, lo que significa que ambas 
zonas están igualmente especializadas en el sector en estudio. Si la participación de la zona $\mathrm{j}$ es mayor que la de referencia, el IES será mayor a 1 , e indica que la zona $j$ está más especializada que la zona de referencia en dicho sector. Finalmente, si el IES resultara menor a 1, significaría que la región en estudio está relativamente poco especializada en ese sector. En el extremo, el mínimo valor que puede tomar este índice es 0 , revelando ausencia de especialización de la zona en el sector elegido (participación del sector igual a cero).

- Índice de concentración de Herfindahl-Hirschman (HHI): cuyo origen se encuentra en la Teoría de la Organización Industrial, es utilizado en el análisis del desarrollo regional con el objetivo de medir el grado de concentración de la producción de una zona entre los diferentes sectores. Su calcula de la siguiente forma:

$$
I H H=\sum_{i}\left(\frac{x_{i j}}{x_{j}}\right)^{2}
$$

donde $x_{i j}$ es el $V A B$ del i-ésimo sector de la región $j$ y $x$ j el VAB total de la zona $j$. Un valor del índice igual a 1 indica que la zona se encuentra completamente especializada en un único sector, mientras que si toma valores cercanos a $1 / \mathrm{n}$ (siendo $\mathrm{n}$ el número de sectores considerados) implica que la producción de la zona analizada se encuentran muy diversificadas entre los distintos sectores.

Se planteó como hipótesis que la política macroeconómica nacional, que favoreció a los sectores exportadores a lo largo de casi toda la última década, debería haber beneficiado a los territorios menos desarrollados de Mendoza, especializados en el sector agrario, contribuyendo de este modo a la disminución de las disparidades. Para comprobarla, se calcularon índices de especialización económica y se vincularon los resultados obtenidos con los correspondientes al análisis de convergencia. La figura 4 demuestra una fuerte relación inversa entre nivel de actividad económica y especialización agropecuaria. Para una rápida comprensión se presentan gráficamente las relaciones entre ranking de indicadores. Se señala con 1 el departamento con mayor IES agropecuario y mayor PBGpc. 
Figura 4. Relación entre PBG Departamental e Índice Simple de Especialización Agropecuaria

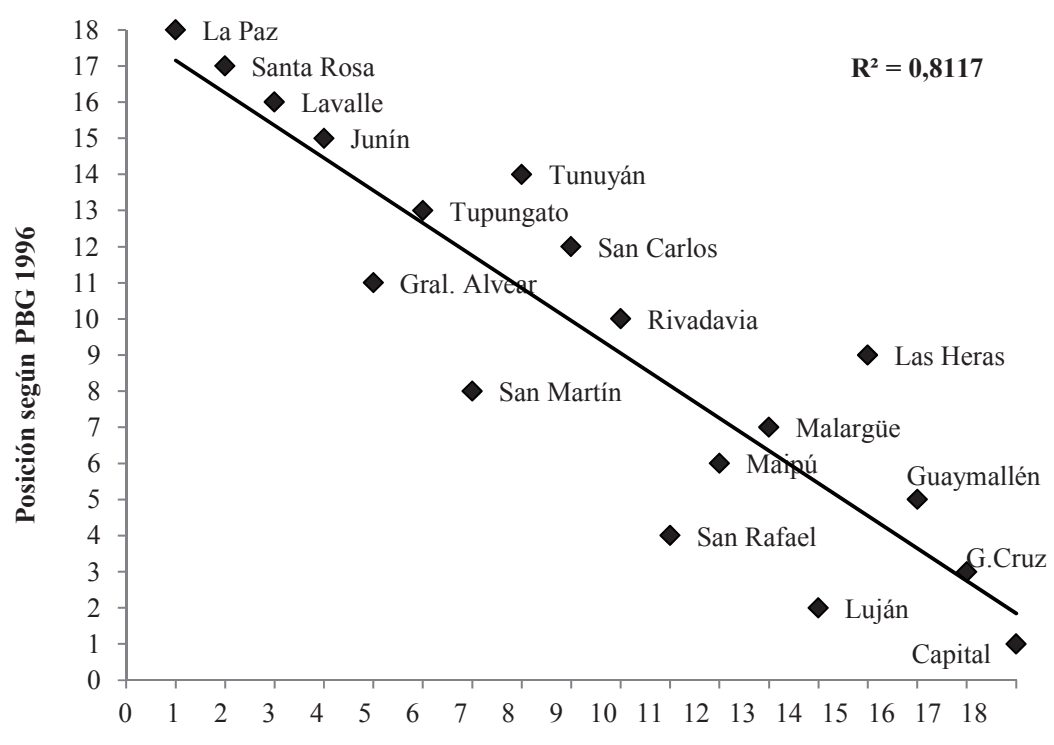

Posición según IES agrícola prov. 1996

Fuente: elaboración propia.

\section{RESULTADOS OBTENIDOS}

En esta primera parte se analiza la evolución de las desigualdades de PBGpc entre los departamentos de Mendoza, durante el período 1996-2012, se compara con la etapa iniciada en 2003, caracterizada por una política macroeconómica más estable, y se interpretan los resultados obtenidos.

\section{III.1. Disparidad}

En esta sección se presentan los valores estimados del Coeficiente de Williamson, normalizados considerando el año 1996 como base $(1996=100)$. La evolución de este indicador demuestra que las disparidades departamentales se intensificaron a lo largo del período tomando, en promedio, valores más altos en el 
subperíodo 2003-2012 que en el lapso completo (figura 5). Por otra parte, la disparidad disminuye en años de crisis, creciendo en años de recuperación económica, conducta que podría deberse a la mayor capacidad de las regiones ricas para adaptarse y, en consecuencia, beneficiarse con las nuevas condiciones.

Figura 5. Coeficiente de Williamson. Período 1996 a 2012

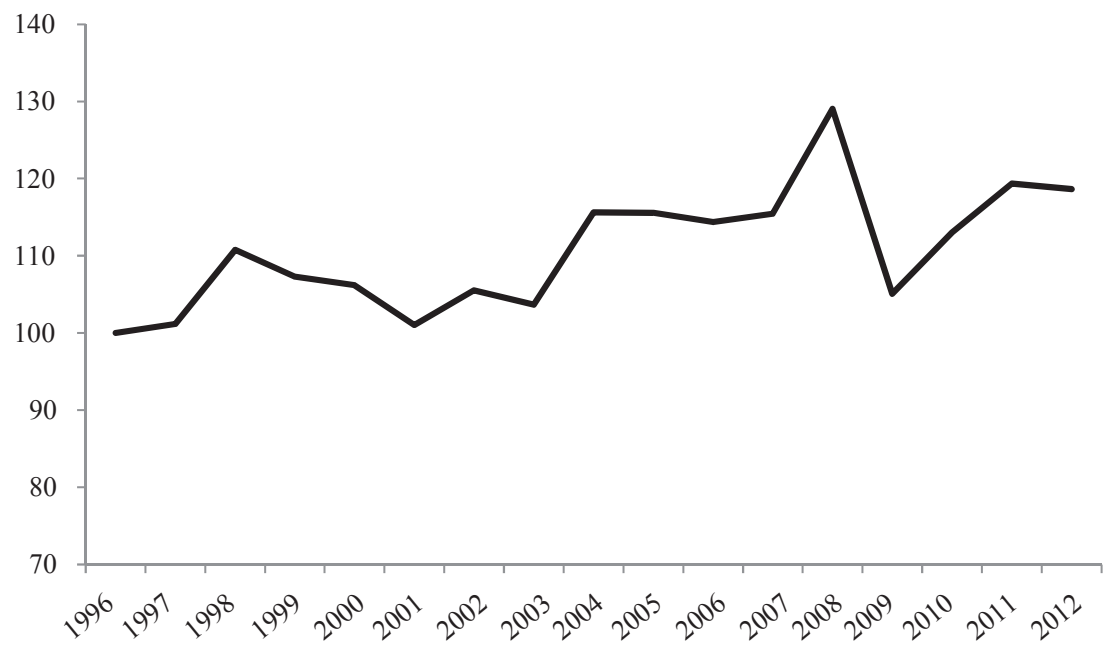

Fuente: elaboración propia.

III.2. Brechas de crecimiento

Se calculó en cuánto se debería haber incrementado la tasa de crecimiento real correspondiente a cada departamento para nivelarse, al final del período, con el departamento con mayor $P B G p c$ y en cuánto debería haberse incrementado esta misma tasa para alcanzar el $P B G p c$ promedio provincial. Para cada período y departamento se calculó la tasa real de crecimiento anual (TCA), la tasa de crecimiento estimada (TCE) requerida para igualar el $P B G p c$ más alto al final de cada período y la tasa de crecimiento promedio $(T C P)$ necesaria para alcanzar el PBG/ habitante promedio de cada período. Mediante la diferencia entre estas dos últimas tasas y la $T C A$ se calculan las dos brechas. Los resultados se presentan en la tabla1 del apéndice. 
Dado que a lo largo de estos años se implementaron programas económicos y políticos con diferencias importantes, se decidió presentar la evolución de estas magnitudes (TCE-TCA y TCP-TCA) durante los subperíodos 1996-2003 y 2003-2012. Una menor pendiente indica que los departamentos más alejados de la primera posición o del promedio necesitan incrementar menos su tasa de crecimiento para alcanzar esas posiciones.

Figura 6. Brechas en las tasas de crecimiento. TCE-TCA y TCP-TCA
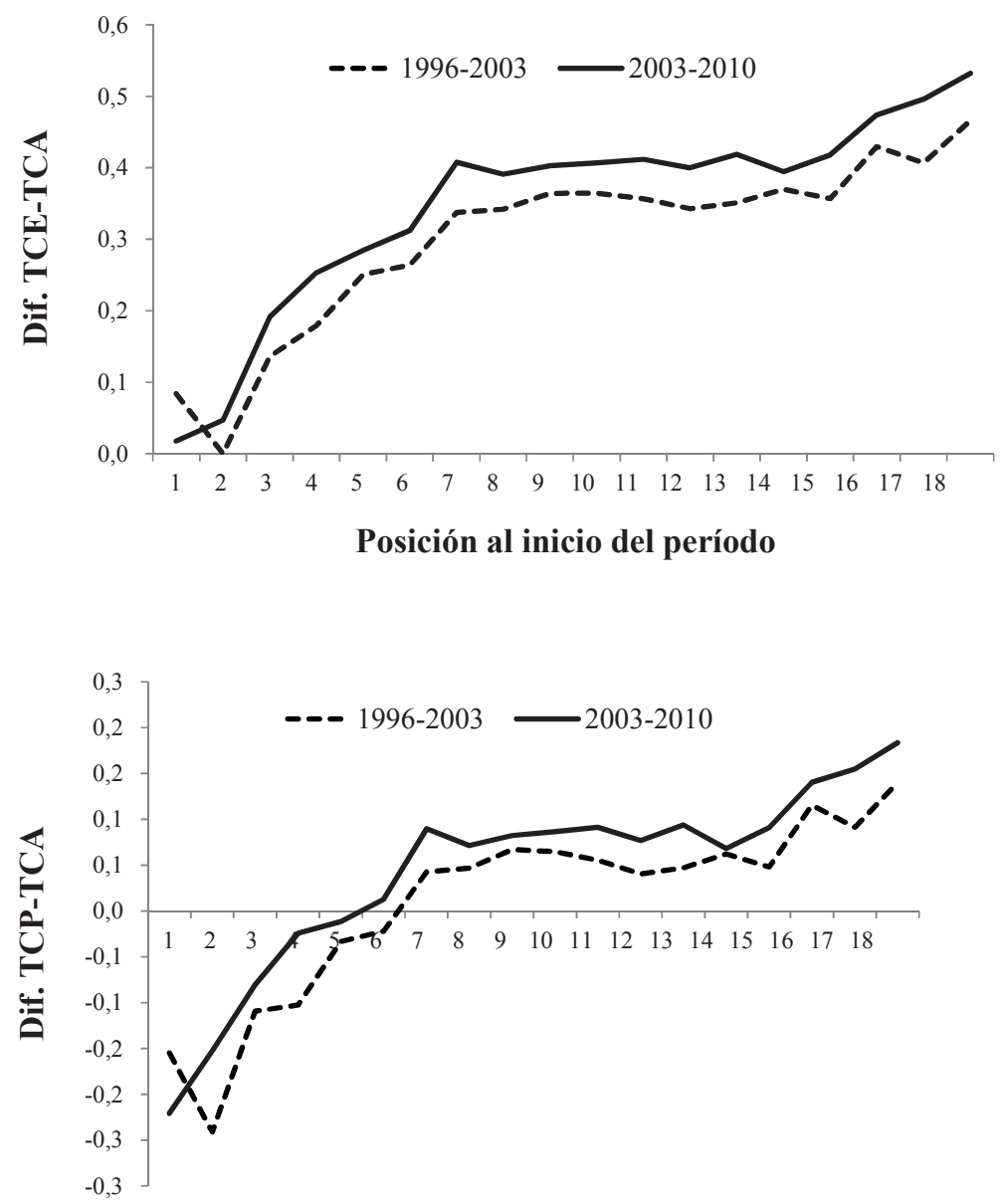

Posición al inicio del período

Fuente: elaboración propia. 
Siendo las ecuaciones de regresión determinadas:

$y=0,023 x+0,125\left(R^{2}=0,781\right)$ corresponde al período 1996-2003

$y=0,017 x+0,123\left(R^{2}=0,781\right)$ corresponde al periodo 1996-2003

$y=0,020 x+0,102\left(R^{2}=0,788\right)$ corresponde al período 2003-2012

$y=0,016 x+0,147\left(R^{2}=0,762\right)$ corresponde al período 2003-2012

En ambas figuras se observa que las brechas, tanto respecto del departamento más rico como respecto del promedio provincial, son mayores en el segundo subperíodo. Se aprecia que la curva roja está por encima de la azul para todos los puntos, lo que significa que la afirmación es válida para todos los departamentos. Se observa que los territorios intermedios (posiciones 7 a 15) mantienen aproximadamente las mismas brechas en ambos períodos. En cambio, en los extremos las diferencias son mayores. Así, al departamento ubicado en la segunda posición, pasar a la tercera le resulta más costoso que retrasarse una posición en el tramo intermedio (del octavo al noveno lugar, por ejemplo). Finalmente, retrasarse un lugar en las últimas posiciones vuelve tener mayor costo.

\section{III.3. Sigma y Beta Convergencia}

A continuación, se exponen los resultados de los dos análisis de convergencia propuestos por Sala-i-Martín. En primer lugar, como ya se explicó, el concepto de $\beta$-convergencia se define como la existencia de una relación inversa entre tasa de crecimiento de la renta y nivel inicial de la misma, para un conjunto determinado de economías. La ecuación a estimar es la siguiente:

$$
\ln \left(y_{i, 2012}\right)-\ln \left(y_{i, 1996}\right)=\alpha+\beta \ln \left(y_{i, 1996}\right)+v_{i}
$$

donde es el PBGpc del departamento en el período . Al estar las variables en logaritmos, la diferencia en el miembro izquierdo de la ecuación es aproximadamente igual a la tasa de crecimiento del departamento en el período bajo estudio. Para que se compruebe la hipótesis de $\beta$-convergencia, el coeficiente $\beta$ debería resultar menor a cero. Se estiman los coeficientes obtenidos mediante el método de Mínimos Cuadrados Ordinarios y la ecuación estimada es:

$$
\ln \left(y_{i, 2012}\right)-\ln \left(y_{i, 1996}\right)=0.396+0.114 \ln \left(y_{i, 1996}\right)
$$

El coeficiente $\beta$ resulta positivo, aunque no significativamente distinto de cero, ni siquiera a un nivel de significancia del $10 \%$. Por su parte, el coeficiente $\alpha$ resulta significativamente distinto de cero al $1 \%$. Además, el $R^{2}$ de la estimación resultó 
igual a 0.117 , lo cual indica que el modelo que incluye la variable explicativa (el logaritmo de la renta en 1996) no difiere significativamente del modelo explicado solo con una constante.

La relación analizada también puede representarse gráficamente, advirtiéndose que no se observa relación entre nivel inicial de renta per cápita y su tasa de crecimiento. Por todo ello, se rechaza la hipótesis de existencia de relación entre el logaritmo del PBG por habitante y su tasa de crecimiento.

Figura 7. $\beta$ convergencia. Período 1996 a 2012

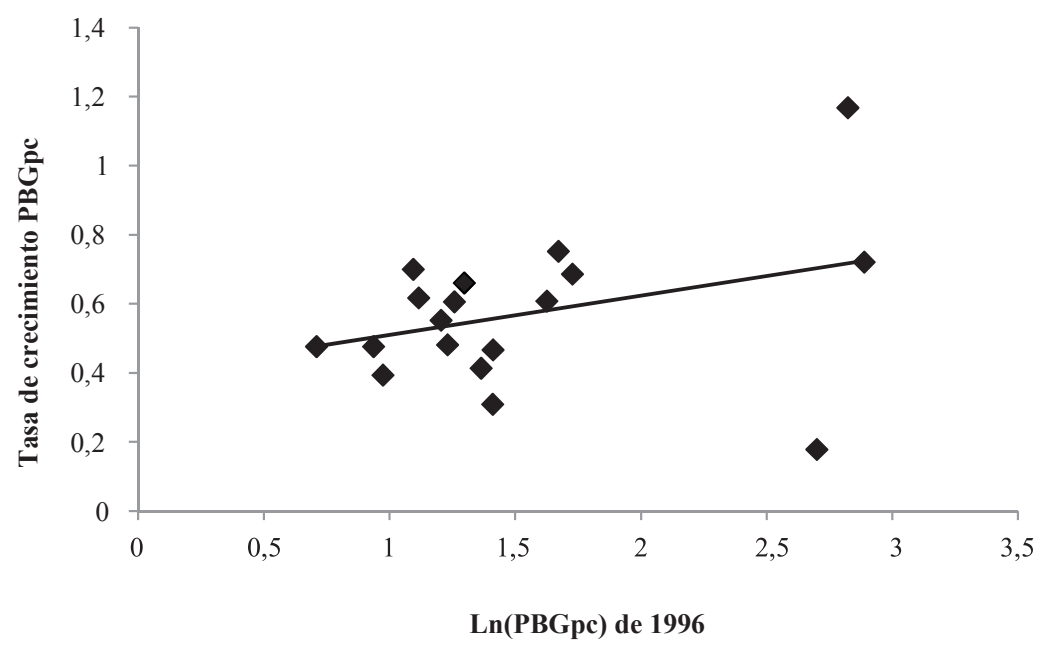

Fuente: elaboración propia.

En segundo lugar, se muestran los resultados del análisis de $\sigma$-convergencia que, como se explicó, es el concepto según el cual la dispersión del PBGpc entre economías tiende a reducirse en el tiempo. Como medida de dispersión se utiliza la fórmula de $C v \sigma_{t}$ ya explicada en la sección de metodología.

Como se observa en la figura 8, existe una tendencia suavemente creciente, con fluctuaciones importantes, si se analiza el período completo, reforzando la conclusión de inexistencia de un proceso de convergencia. Se pueden establecer cinco etapas bastante marcadas. Entre 1996 y 1998, época de expansión económica, el índice se incrementa casi un $11 \%$. A partir de entonces decrece, hasta llegar a 2001. Continúa una etapa de crecimiento, con algunas fluctuaciones, hasta 
2008. El año 2009 marca una importante caída, que se revierte en los años posteriores, a ritmo decreciente.

Figura 8. $\sigma$ convergencia. Período 1996 a 2012

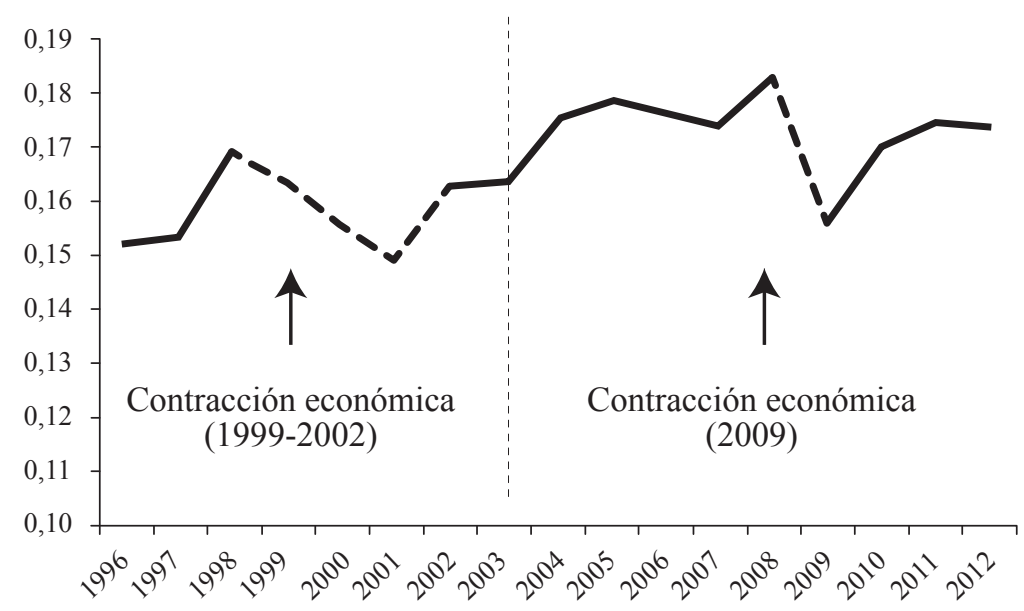

Fuente: elaboración propia.

Una de las posibles explicaciones de la ausencia de $\beta$-convergencia observada, junto con el aumento en la dispersión de los ingresos per cápita departamentales (o divergencia en el sentido sigma) se relaciona con los niveles de PBGpc de los departamentos en estudio. Según Williamson (1965), la desigualdad regional tiene forma de "U invertida", aumentando significativamente con el crecimiento económico a bajos niveles de PBGpc y descendiendo una vez alcanzado un máximo. Es decir, se tiende a la convergencia. En otras palabras, podría estar sucediendo que el PBGpc provincial no ha alcanzado aún el nivel suficiente para que se generen las condiciones económicas necesarias para la existencia de convergencia en los ingresos per cápita departamentales ${ }^{4}$.

4 No obstante, la tesis de Williamson ha sido estudiada ampliamente en la literatura económica, generando posturas diversas, en cuanto a que si con un nivel de desarrollo alto, las desigualdades divergirán o convergerán. Ante tal situación, se han presentado dos perspectivas: i) divergencia regional, expuesta por Gunnar Myrdal (considerada como la visión de izquierda); y ii) convergencia regional, expuesta por John Friedmann y Clyde Weaver (considerada como la visión de derecha). Para Myrdal, siempre existirá la relación centro-periferia (sin la intervención del Estado), ya que los mecanismos del mercado favorecerán la agravación de desigualdades. Factores como migración, movimientos de capitales y comercio dejan a las regiones más pobres al perder a los mejores elementos para su desa- 


\section{III.4 Convergencia y especialización económica}

Dado que los departamentos de menor ingreso por habitante son también los de mayor especialización agropecuaria y considerando la política macroeconómica nacional a partir del año 2003, marcada por un tipo de cambio competitivo, se consideró que podría haber favorecido relativamente más a aquellos departamentos dedicados a especialmente a la producción agropecuaria. Si esto fuera cierto debería observarse una relación positiva entre el nivel relativo de especialización en sector agropecuario y el crecimiento del PBG, al menos a partir del año 2003. A continuación se presenta gráficamente esta relación para el período completo y para el subperíodo 2003-2012 (Figura 9). En el eje de abscisas se ubican los departamentos según su posición en el ranking de especialización agropecuaria, medida a través del IES y en el eje de ordenadas de acuerdo su posición en el ranking de crecimiento medio anual. Los valores numéricos pueden observarse en la tabla 2 del Apéndice.

Tanto en la figura 9a), que corresponde al período completo, como en la $9 \mathrm{~b}$ ), del segundo subperíodo, se advierte un buen número de territorios que han evolucionado de modo similar, con independencia de su especialización productiva. Debe destacarse que los departamentos de Capital y Godoy Cruz, que no desarrollan actividad agropecuaria (IES de valor cero), ocupan el primer y segundo lugar en crecimiento, ganando posición relativa durante este segundo subperíodo. Por otra parte, Lavalle, Santa Rosa, La Paz y Junín, los cuatro territorios de mayor especialización agrícola, presentan bajas tasas de crecimiento. Por todo ello, se rechaza la hipótesis de investigación planteada.

\section{III.5 Análisis por regiones}

Como se mencionó anteriormente, la fuerte interrelación entre departamentos vecinos y, en especial, la libre movilidad de los factores de producción (fundamentalmente el trabajo), hacen que los límites departamentales sean difusos, lo cual siembra la duda sobre la precisión del PBGpc como medida del ingreso por habitante. Por ejemplo, la gente migra desde territorios de mayor densidad de población a otros cercanos, menos urbanos, que le permiten mantener su actividad económica y disfrutar de mejor calidad de vida. Esto hace que las tasas de

rrollo, y que él llama factores de estancamiento. En lo referente a la visión de Friedman y Weaver, los autores consideran que existen factores que harán que tiendan a disminuir las disparidades regionales, a partir de una integración económica. Sostienen que los mecanismos libres del mercado harán restablecer una nivelación de las diferencias entre personas y entre regiones 
Figura 9. Relación entre especialización agropecuaria y crecimiento del ingreso por habitante

\section{9 a).Período 1996-2012}

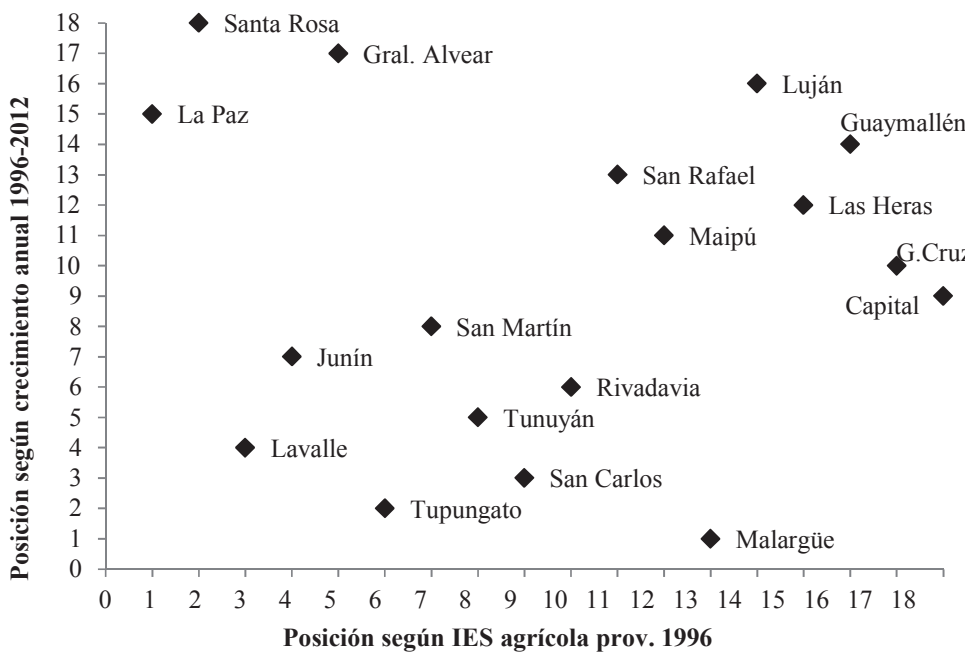

9 b). Período 2003-2012

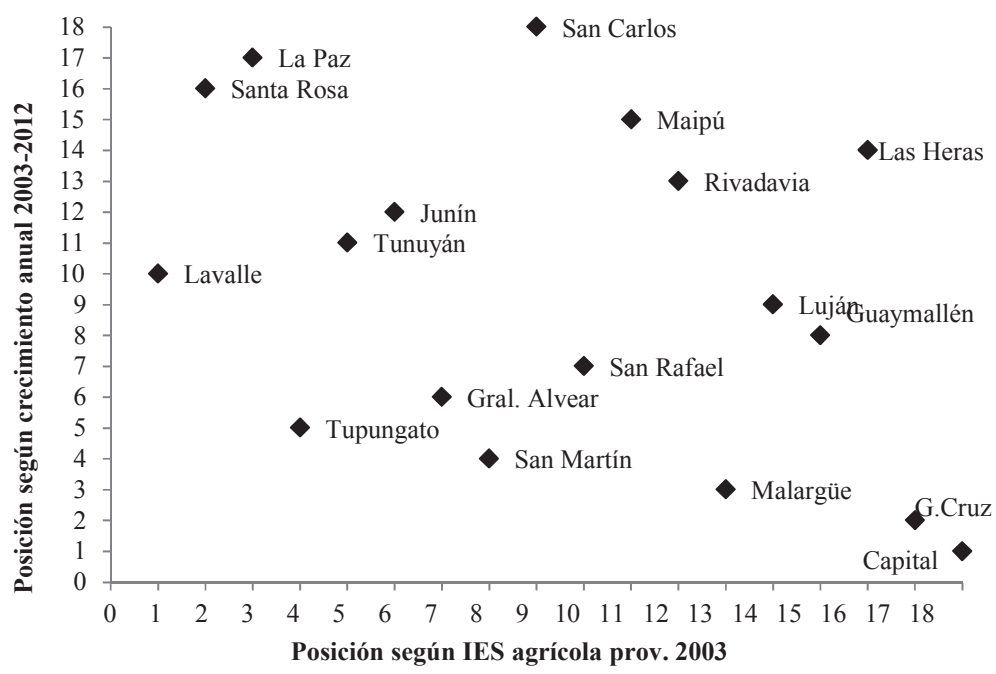

Fuente: elaboración propia. 
crecimiento poblacional sean menores en los primeros, situación que influye en la estimación del PBGpc.

Como una posible solución a este problema, se calcularon los coeficientes de disparidad y dispersión para las cinco regiones de la provincia: Gran Mendoza, Este, Noreste, Valle de Uco y Sur ${ }^{5}$. Se considera que la misma es una solución imperfecta al planteo anterior, ya que si bien la cercanía geográfica de los departamentos incluidos en una misma región resuelve el problema de la movilidad del factor trabajo, los resultados obtenidos serán promedios de los verdaderos valores observados. A su vez, la cantidad de unidades de análisis se reduce drásticamente, impidiendo la realización de análisis para los cuales se requiere una mayor cantidad de observaciones, tales como $\beta$ - convergencia o la estimación de las brechas de crecimiento. Se tomó el año 1996 como base. Como se puede observar, durante el período 2003-2012 el coeficiente de Williamson es menor que en el año base para todos los años, con excepción del año 2008. Pese a ello, no se aprecia una tendencia definida, a diferencia de lo que ocurría en el análisis a nivel departamental, en el cual existía una clara tendencia creciente en la evolución del coeficiente.

A pesar de que los resultados son menos claros que en el análisis anterior (y esto se debe a que los coeficientes representados en este caso son promedios a nivel regional), aún se aprecia la reducción de la disparidad en años de crisis (1998-2001 y 2009) y un aumento de la misma en los años de recuperación económica (2003-2007), como sucedía en el análisis a nivel departamental.

En cuanto a $\sigma$-convergencia, cuando se realizan los cálculos teniendo en cuenta el PBGpc regional, se obtiene una tendencia en forma de U. A grandes rasgos, se observa una disminución de la dispersión en el primer subperíodo en estudio, revirtiéndose la tendencia con la recuperación económica del año 2003.

\section{CONCLUSIONES}

Un posible motivo de falta de convergencia surge del hecho de que las economías departamentales pueden tener diferentes estados estacionarios, de modo que, a pesar de que cada una está tendiendo al suyo propio, el resultado de este método

5 La composición de las regiones es la siguiente: Gran Mendoza (Capital, Guaymallén, Godoy Cruz, Las Heras, Luján de Cuyo y Maipú), Este (Rivadavia, San Martín y Junín), Noreste (La Paz, Lavalle y Santa Rosa), Valle de Uco (Tunuyán, Tupungato y San Carlos), Sur (General Alvear, Malargüe y San Rafael). 
de análisis indicará ausencia de convergencia ${ }^{6}$. La especialización productiva y el ritmo de innovación tecnológica son algunos de los factores que pueden determinar diferencias en el estado estacionario. Como se observa en la tabla 2 del apéndice, diferentes territorios tienen distinta especialización productiva y diferente grado de concentración de actividades. Por otra parte, debe tenerse presente que a lo largo de las dos últimas décadas, la actividad vitivinícola provincial ha sido una importante receptora de inversiones, distribuidas en el territorio de modo desigual. Así, la Región del Valle de Uco, integrada por los departamentos de Tupungato, Tunuyán y San Carlos ha recibido inversión extranjera directa de moderna tecnología, en una magnitud superior al resto de los departamentos agrícolas.

Una posibilidad para testear la existencia de distintos estados estacionarios $y$, bajo este supuesto, poner en estudio nuevamente la posibilidad de $\beta$-convergencia sería aplicar el método de las regresiones múltiples. La idea de este método es efectuar una regresión con los datos de sección cruzada del crecimiento sobre la renta inicial, manteniendo constante un cierto número de variables adicionales (que actúan de proxy del estado estacionario). De este modo, si se observara una relación negativa entre $\mathrm{PBGpc}$ inicial y tasa de crecimiento, entonces se confirmaría la hipótesis de convergencia Beta. Algunas de las variables que en la literatura empírica se han utilizado para representar el estado estacionario de cada región son gasto público, transferencias intergubernamentales, especialización de la región en el sector agrícola, variables representativas del capital humano, infraestructura, entre otras.

Resulta interesante plantear algunas razones microeconómicas que podrían explicar, al menos parcialmente, los resultados obtenidos. En efecto, se ha comprobado:

- la existencia de asimetrías de eficiencia productiva dentro del eslabón agrícola de la cadena agroindustrial, siendo las explotaciones de mayor tamaño las que han demostrado mayor eficiencia productiva y mayor capacidad de adaptación a los cambios en las demandas nacional e internacional (Pasteris \& Palazzo, 2007);

- condiciones que facilitarían el ejercicio de poder de mercado en las compras por parte de los eslabones industrial y/o de comercialización de la cadena (Pasteris \& Garriga Suárez, 2007) y

- asimetrías en los atributos que influyen sobre la probabilidad de exportar y, en general, de aprovechar los beneficios del cluster vitivinícola por parte de las firmas (Pasteris \& González, 2011).

6 Sala-i-Martín (1990), Barro y Sala-i-Martín (1991, 1992a, 1992b) y Mankiw, Romer y Weil (1992). 
Estas características de la cadena agroindustrial influyen en la distribución de la renta entre los eslabones que las integran, de manera que, por una parte, el eslabón agrícola recibe solo una pequeña proporción del valor agregado total $\mathrm{y}$, por otra, su distribución en el territorio no es pareja, ya que depende de la localización de las firmas en mejores condiciones de aprovechar las ventajas del crecimiento. En la medida en que esta localización no corresponda a los territorios de mayor especialización agropecuaria (y menor desarrollo relativo), esta circunstancia contribuirá a rechazar la hipótesis planteada.

Finalmente, se destaca que una continuidad deseable de esta investigación está dada por la comparación de estos resultados con el análisis de convergencia medida a través de indicadores de salud, nivel de educación, condiciones de vivienda, seguridad, y otros, como variables proxy del bienestar. En este sentido, cabe citar a Gilbert y Goodman (1976) quienes sostuvieron que “(...) la convergencia regional de ingresos puede estar asociada con logros muy pequeños (e incluso un descenso) en los ingresos de los grupos más pobres de la sociedad, con un empeoramiento en la distribución del ingreso en las regiones más pobres". Hasta el momento de finalización de este documento, no estaba disponible la información del Censo Nacional de Población 2010, necesaria para construir estos indicadores. 


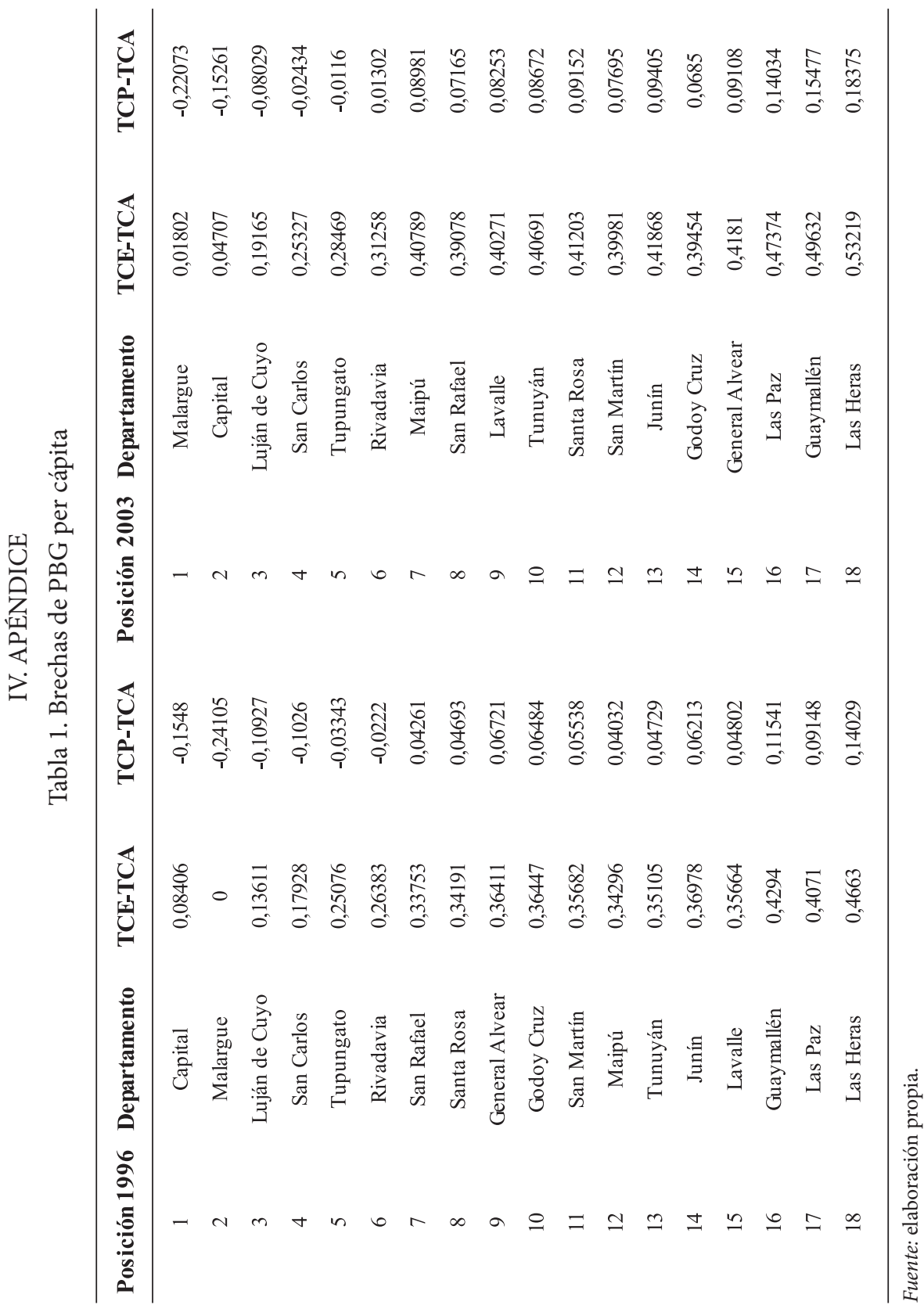




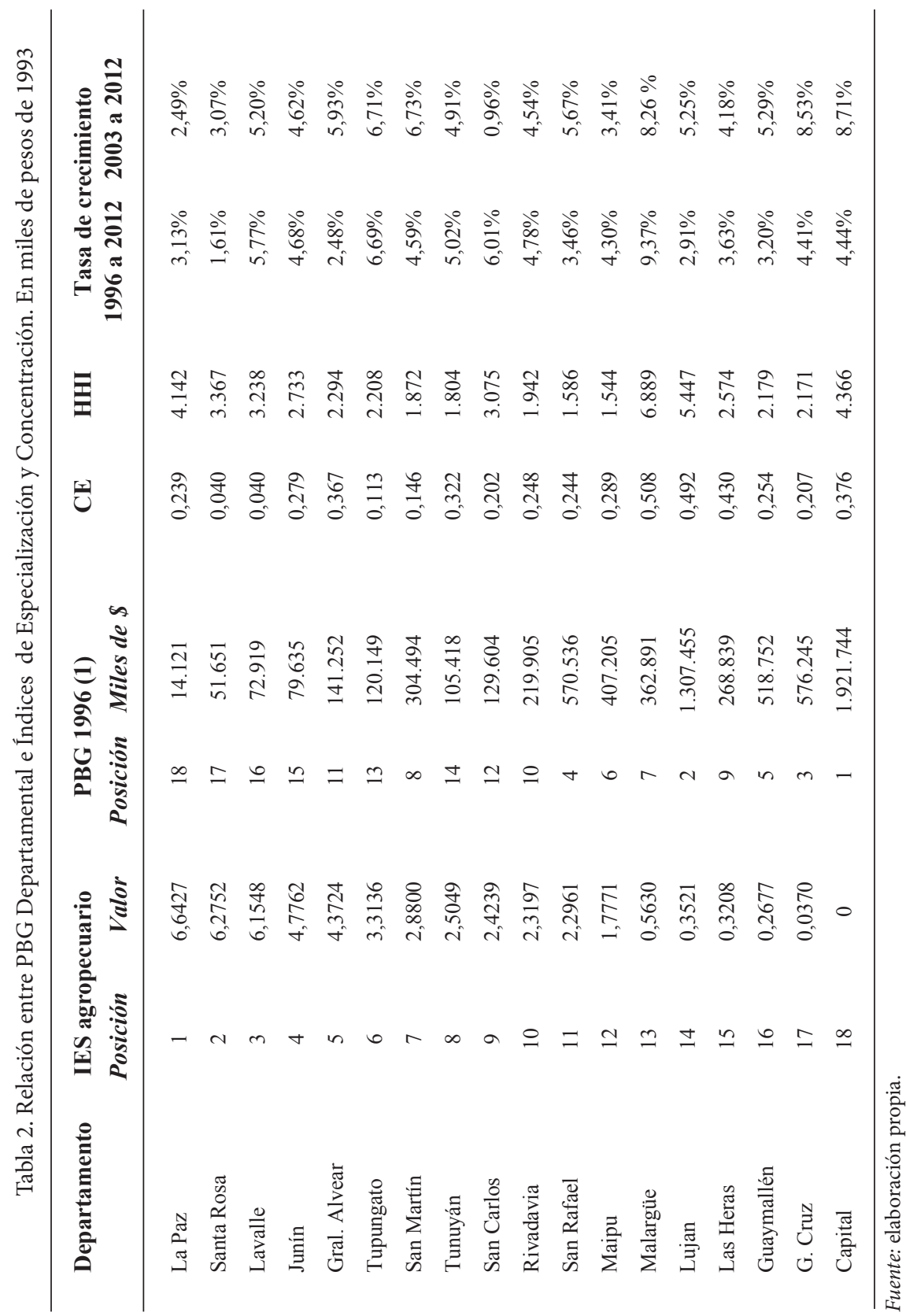




\section{REFERENCIAS BIBLIOGRÁFICAS}

Bignone, F. (2011). Convergencia y Determinantes del Crecimiento en las Provincias Argentinas (Tesis de pregrado). Universidad Nacional de Cuyo, Mendoza, Argentina.

Capello, M., Figueras, A., Freille, S. \& Moncarz, P. (2011). Transferencias Fiscales a Provincias y Convergencia en Crecimiento y Bienestar Regional. XXIII Seminario Regional De Política Fiscal, CEPAL, Chile. Recuperado de http://www.cepal.org/ilpes/noticias/paginas/1/41751/capello_figueras_freille_ moncarz trans fiscales doc.pdf el 25/02/2016.

Easterly, W. (2003). En busca del crecimiento. Andanzas y tribulaciones de los economistas del desarrollo. Barcelona, España: Antoni Bosch ed.

Figueraz, A. J., Arrufat J. L. \& Regis, P. J. (2003). El fenómeno de la convergencia nacional: una contribución. Anales de la Asociación Argentina de Economía Política. Recuperado de http://www.aaep.org.ar/anales/works/ works2003/Figueras_Arrufat_Regis.pdf el 25/02/2016.

Garrido, N., Marina, A. \& Sotelsek, D. (2001). Crecimiento y convergencia: un ejercicio empírico sobre las regiones españolas y las provincias argentinas. Anales de la Asociación Argentina de Economía Política.

Garrido, N., Marina, A. \& Sotelsek, D. (2001). Convergencia económica en las provincias argentinas (1970-1995). Estudios de Economía Aplicada, 20 (2). Recuperado de http://www.redalyc.org/pdf/301/30120207.pdf el 25/02/2016.

Gilbert, A. \& Goodman, D. (1976). Desigualdades regionales de ingreso y desarrollo económico: un enfoque crítico. Revista Latinoamericana de Estudios Urbano Regionales, 5 (13), 79-101.

Guilló, M. (2009). Análisis económico de los departamentos de Mendoza (Tesis de pregrado). Mendoza, Argentina: Universidad Nacional de Cuyo,

Hernández Aragón, J. (2006). Las disparidades regionales: ¿Hacia la convergencia o divergencia regional? Contribuciones a la Economía, 3. Recuperado de http://www.eumed.net/ce/2006/jha.htm el 25/02/2016.

Kemnitz, T. (2013). Convergencia y Especialización en los Departamentos de Mendoza (Tesis de pregrado). Mendoza, Argentina: Universidad Nacional de Cuyo.

Lira Cossio, L. (2003). La Cuestión Regional y Local en América Latina. ILPES Serie Gestión Pública, 44. Recuperado de http://repositorio.cepal.org/bitstream/handle/11362/7298/S0311857_es.pdf?sequence=1 el 25/02/2016.

Marina, A. (1998). Convergencia económica en la Argentina. ¿Qué nos dice la evidencia empírica? Anales de la Asociación Argentina de Economía 
Política. Recuperado de http://www.aaep.org.ar/anales/works/works1998/ MARINA.PDF el 25/02/2016.

Medawar, A., Pasteris, E., Carretero, M., Perlbach, I. \& Calderón, M. (2013). Estimación del PBG de Mendoza y sus departamentos. Años 1991 a 2010. FCE-UNCuyo y DEIE Mendoza.

Pasteris, E. \& Palazzo, R. (2007). Estructura-Conducta-Resultados en mercados agroindustriales. El rol de la defensa de la competencia y de las políticas industriales activas. Anales de la $V^{a}$ Conferencia de Investigación en Entrepreneurship en América Latina y XII ${ }^{a}$ Reunión Anual de la Red PyMEs del MERCOSUR.

Pasteris, E. \& Garriga Suárez, P. (2007). Asimetrías entre precios mayoristas y minoristas. El caso del vino común en Argentina. Anales de la Asociación Argentina de Economía Política.

Pasteris, E. \& González, R. (2011). Inserción internacional de la industria vitivinícola de Mendoza, al iniciarse el siglo XXI, en Vinos y competitividad agroindustrial: un largo camino, compilado por Ana María Mateu. Mendoza, Argentina: Ed. Inca.

Pasteris, E., Jan Casaño, R., Salvador P. \& Suoni, A. (2013). Estimación del PBG de Mendoza y sus departamentos. Años 2011 a 2013. Consejo Federal de Inversiones.

Pasteris, E., Farreras, V., Salvador, P., Garriga Suárez, P. \& Kuschnaroff, V. (2012). Matriz productiva provincial. Universidad Nacional de Cuyo.

Platino, M. (2013). Análisis de la Desigualdad y Convergencia en los niveles de Actividad Industrial en la Provincia de Santa Fe, UNR-CONICET.

Porto, G. (1994). Convergencia y Política Económica. Algunos resultados para las Provincias Argentinas. Anales de la Asociación Argentina de Economía Política. Recuperado de http://www.aaep.org.ar/anales/works/works1994/ PortoGuido.pdf_el 25/02/2016.

Quinteros, M. (2009). Convergencia Económica en las Provincias Argentinas entre 1953 y 2003 (Tesis de pregrado). Universidad del Salvador, Mendoza. Recuperado de http://www.usal.edu.ar/archivos/eco/docs/quinteros.pdf el 25/02/2016.

Red De Observatorios De Cuyo (2014). Evolución de las condiciones socioeconómicas del territorio mendocino. Serie Diagnóstico y Perspectivas Regionales, Documento Nro. 4.

Romer, D. (1996). Macroeconomía avanzada. Estados Unidos: McGraw-Hill.

Russo, J. L. \& Ceña Delgado, F. (2000). Evolución de la Convergencia y Disparidades provinciales en Argentina. Revista de Estudios Regionales, 57, 151-173. Recuperado de http://www.revistaestudiosregionales.com/ documentos/articulos/pdf661.pdf el 25/02/2016. 
Sala-i-Martín, X. (2000). Apuntes de crecimiento económico. Barcelona, España: Antoni Bosch.

Utrera, G. \& Koroch J. (1998). Convergencia: evidencia empírica para las provincias argentinas (1953-1994). Anales de la Asociación Argentina de Economía Política. Recuperado de http://www.aaep.org.ar/anales/works/ works 1998/utera_koroch.pdf el 25/02/2016.

Williamson, J. G. (1965). Regional inequality and the process of national development: a description of the patterns. Economic Development and Cultural Change, 13(4), 1-84. Recuperado de http://www.jstor.org/ stable/1152097?seq=1\#page_scan_tab_contents el 25/02/2016.

(C) 2016 por los autores; licencia otorgada a la Revista Estudios Económicos. Este artículo es de acceso abierto y distribuido bajo los términos y condiciones de una licencia Atribución-No Comercial 3.0 Unported (CC BY-NC 3.0) de Creative Commons. Para ver una copia de esta licencia, visite http://creativecommons.org/ licenses/by-nc/3.0/ 
